\title{
DNA-based stable isotope probing coupled with cultivation methods implicates Methylophaga in hydrocarbon degradation
}

\author{
Sara Mishamandani ${ }^{1}$, Tony Gutierrez ${ }^{1,2 *}$ and Michael D. Aitken ${ }^{1}$ \\ 1 Department of Environmental Sciences and Engineering, Gillings School of Global Public Health, University of North Carolina, Chapel Hill, NC, USA \\ ${ }^{2}$ Centre for Marine Biodiversity and Biotechnology, School of Life Sciences, Heriot-Watt University, Edinburgh, UK
}

\section{Edited by:}

Andreas Teske, University of North Carolina at Chapel Hill, USA

\section{Reviewed by:}

Kathleen Scott, University of South Florida, USA

Molly C. Redmond, University of North Carolina at Charlotte, USA

\section{*Correspondence:}

Tony Gutierrez, School of Life

Sciences, Heriot-Watt University,

John Muir building, Edinburgh EH14

$4 A S, U K$

e-mail: tony.gutierrez@hw.ac.uk
Marine hydrocarbon-degrading bacteria perform a fundamental role in the oxidation and ultimate removal of crude oil and its petrochemical derivatives in coastal and open ocean environments. Those with an almost exclusive ability to utilize hydrocarbons as a sole carbon and energy source have been found confined to just a few genera. Here we used stable isotope probing (SIP), a valuable tool to link the phylogeny and function of targeted microbial groups, to investigate hydrocarbon-degrading bacteria in coastal North Carolina sea water (Beaufort Inlet, USA) with uniformly labeled $\left[{ }^{13} \mathrm{C}\right] n$-hexadecane. The dominant sequences in clone libraries constructed from ${ }^{13} \mathrm{C}$-enriched bacterial DNA (from $n$-hexadecane enrichments) were identified to belong to the genus Alcanivorax, with $\leq 98 \%$ sequence identity to the closest type strain-thus representing a putative novel phylogenetic taxon within this genus. Unexpectedly, we also identified ${ }^{13} \mathrm{C}$-enriched sequences in heavy DNA fractions that were affiliated to the genus Methylophaga. This is a contentious group since, though some of its members have been proposed to degrade hydrocarbons, substantive evidence has not previously confirmed this. We used quantitative PCR primers targeting the 16S rRNA gene of the SIP-identified Alcanivorax and Methylophaga to determine their abundance in incubations amended with unlabeled $n$-hexadecane. Both showed substantial increases in gene copy number during the experiments. Subsequently, we isolated a strain representing the SIP-identified Methylophaga sequences (99.9\% 16S rRNA gene sequence identity) and used it to show, for the first time, direct evidence of hydrocarbon degradation by a cultured Methylophaga $\mathrm{sp}$. This study demonstrates the value of coupling SIP with cultivation methods to identify and expand on the known diversity of hydrocarbon-degrading bacteria in the marine environment.

Keywords: hydrocarbon degradation, stable isotope probing, Methylophaga, Alcanivorax, $n$-hexadecane, marine environment

\section{INTRODUCTION}

Hydrocarbon-degrading bacteria comprise an important component to the total microbial diversity in the marine environment, contributing significantly to the degradation and ultimate removal of hydrocarbons from the marine water column and sediment. Considering the enormous volumes of hydrocarbons that enter the oceans each year through natural seepage, anthropogenic activities and other sources, the fact that the sea surface is not covered in a layer of oil is largely due to the presence and activities of these types of bacteria. Species of hydrocarbondegrading bacteria, belonging to over 20 genera and distributed across some of the major bacterial Classes (Alpha-, Betaand Gammaproteobacteria; Actinomycetes; Flexibacter-CytophagaBacteroides), have been isolated and described (Floodgate, 1995; Head and Swannell, 1999; Head et al., 2006; Yakimov et al., 2007). To our knowledge, the marine environment is the only place where we find bacteria with the ability to utilize hydrocarbons almost exclusively as a sole source of carbon and energy.
So-called "hydrocarbon specialists", or "obligate oil-degraders", these organisms are ubiquitous in the ocean, often found at $<0.1 \%$ abundance of the total microbial community in seawater, and becoming strongly selected for and increasing to levels constituting up to $90 \%$ of the total microbial community upon exposure to crude oil or its refined petrochemical products (Röling et al., 2002, 2004; Teira et al., 2007). This hydrocarbon-elicited bloom in microbial-expressed hydrocarbon catabolizing potential is one of the ocean's inherent mechanisms to keeping the total load of hydrocarbons to background levels, in-turn helping to mitigate the potential detrimental effects that these chemical pollutants can pose to marine life.

Whilst our knowledge on the diversity of hydrocarbondegrading bacteria in the ocean has progressed, it is far from complete, and novel taxa continue to be discovered. One bacterial genus that has proven contentious with respect to whether any of its members might be capable of degrading hydrocarbons is Methylophaga. Members of this genus are strictly aerobic and 
moderately halophilic, belonging to the Piscirickettsiaceae family in the Gammaproteobacteria, and exhibit an exclusive requirement for $\mathrm{C}_{1}$ sources (methanol, methylamine, dimethylsulfide) as sole growth substrates, with the exception of some species that are also capable of utilizing fructose (Janvier and Grimont, 1995). A few studies have reported the enrichment of Methylophaga spp. in oil-contaminated field samples and in laboratory experiments with oil (Röling et al., 2002; Yakimov et al., 2005; Coulon et al., 2007), but whether those organisms could degrade hydrocarbons was not addressed in those studies. Recently, Vila et al. (2010) isolated a Methylophaga species, designated strain AF3, from a beach impacted by the Prestige oil spill and showed it to grow in a synthetic seawater medium amended with high molecular weight polycyclic aromatic hydrocarbons (PAHs). However, since the medium was also amended with nutritionally-rich LuriaBertani medium, the assumption that strain AF3 could grow on PAHs was unsubstantiated. Hence, compelling evidence showing a Methylophaga species to degrade hydrocarbons remains lacking.

One method that circumvents the requirement to isolate microorganisms in order to assess their metabolic and physiological characteristics is stable isotope probing (SIP). This method has been used successfully on environmental samples to identify a target microbial group(s) based on their ability to perform a specific metabolic process, thereby being able to link the phylogenetic identity of an organism to its function (Dumont and Murrell, 2005). An added advantage of this technique is its ability to identify target members of a microbial community that are not amenable to cultivation in the laboratory. In this study, we investigated hydrocarbon-degrading bacteria in surface seawater on the North Carolina coast using DNA-based SIP with uniformly labeled $\left[{ }^{13} \mathrm{C}\right] n$-hexadecane and identified Alcanivorax and several other taxa, including Methylophaga, in the isolated ${ }^{13} \mathrm{C}$-labeled "heavy" DNA. We subsequently isolated a strain representing this SIP-identified Methylophaga, designated strain SM14, which was found capable of growing on $n$-hexadecane as a sole source of carbon and energy. This work, which couples DNA-SIP with cultivation-based methods, for the first time reveals direct evidence implicating a Methylophaga species with the ability to utilize a hydrocarbon as a growth substrate.

\section{MATERIALS AND METHODS FIELD SAMPLE}

During a field trip 1 mile offshore from the Beaufort Inlet $\left(34^{\circ} 33.42^{\prime} \mathrm{N}, 76^{\circ} 51.06^{\prime} \mathrm{W}\right.$ ), North Carolina, USA on 27 August 2010, ca. $20 \mathrm{~L}$ of surface seawater was collected into preautoclaved polypropylene (Nalgene) bottles and stored at $4^{\circ} \mathrm{C}$. On the following day, ca. $18 \mathrm{~L}$ of the water sample was filtered through $0.2-\mu \mathrm{m}$ Nucleopore filters and the retained biomass resuspended in ONR7a medium (Dyksterhouse et al., 1995) to a total volume of ca. $100 \mathrm{ml}$ to act as the inoculum for use in enrichment, mineralization, degradation and SIP experiments (described below).

\section{SIP INCUBATIONS}

SIP incubations were performed as described previously (Gutierrez et al., 2013). Briefly, 16 125-ml autoclaved glass screwtop Erlenmeyer flasks with caps lined with aluminum foil to prevent the adsorption of hydrocarbons were prepared. Each flask contained $15 \mathrm{ml}$ of ONR7a medium, $1 \mathrm{mg}$ of labeled $\left({ }^{14} \mathrm{C}\right.$ or ${ }^{13} \mathrm{C}$ ) and/or unlabeled $n$-hexadecane, and $5 \mathrm{ml}$ of inoculum. $\left[\mathrm{U}-{ }^{13} \mathrm{C}\right] n$-hexadecane was obtained from Sigma-Aldrich (United States). For SIP, duplicate flasks were prepared with $1 \mathrm{mg}$ of [U- $\left.{ }^{13} \mathrm{C}\right] n$-hexadecane, and a second set of duplicates was prepared with $1 \mathrm{mg}$ of the unlabeled counterpart. To determine the endpoint of each SIP experiment, the mineralization of $\left[\mathrm{U}-{ }^{14} \mathrm{C}\right]$ $n$-hexadecane was measured in triplicate flasks by liquid scintillation counting of ${ }^{14} \mathrm{CO}_{2}$ trapped in $\mathrm{KOH}$-soaked filter paper over time, as described below. An additional set of triplicate flasks was used to monitor the disappearance of unlabeled $n$-hexadecane by gas chromatography-mass spectrometry (GC-MS). Samples were taken periodically from these flasks for DNA extraction and subsequent measurement of the abundance of target organisms (by qPCR as described below) identified through SIP. Triplicate flasks of acid-killed controls $(\mathrm{pH} \leq 2)$ containing unlabeled $n$-hexadecane were prepared by adding $85 \%$ phosphoric acid (ca. $0.7 \mathrm{ml}$ per flask). All flasks were incubated on an orbital shaker $\left(250 \mathrm{rpm} ; 21^{\circ} \mathrm{C}\right)$ in the dark. At the endpoint of each SIP incubation-defined as the time when the extent of mineralization of the ${ }^{14} \mathrm{C}$-labeled $n$-hexadecane began to approach an asymptote-whole DNA from the total volume in the paired flasks amended with the $\left[\mathrm{U}_{-}{ }^{13} \mathrm{C}\right] n$-hexadecane and the corresponding paired set with unlabeled $n$-hexadecane was extracted using the method of Tillet and Neilan (2000).

To monitor the mineralization of $\left[\mathrm{U}^{14} \mathrm{C}\right] n$-hexadecane, each triplicate flask contained the ${ }^{14} \mathrm{C}$-labeled compound to $20,000 \mathrm{dpm}$ and $2.5 \mathrm{mg}$ of unlabeled $n$-hexadecane. Killed controls (in triplicate) were also prepared by adding $85 \%$ phosphoric acid to $\mathrm{pH} \leq 2$ prior to inoculation. For the $\mathrm{CO}_{2}$ trap, a sterile glass test tube $(12 \times 75 \mathrm{~mm})$ containing a piece of filter paper saturated with $60 \mu \mathrm{l}$ of $2 \mathrm{M} \mathrm{KOH}$ was inserted into each flask. The filter paper from each flask was removed daily and the captured ${ }^{14} \mathrm{C}$ from any ${ }^{14} \mathrm{CO}_{2}$ respired was counted on a Packard (Meriden, CT, USA) Tri-Carb liquid scintillation analyser (model 1900TR). The KOH-saturated filter paper from each flask was replaced at each sampling point for the course of the experiment. The percentage of ${ }^{14} \mathrm{C}$ mineralized for $\left[\mathrm{U}_{-}{ }^{14} \mathrm{C}\right] n$-hexadecane was calculated by subtracting the triplicate values for the acidified controls from those of the experimental and then dividing by the total dpm of ${ }^{14} \mathrm{C}$ added.

\section{CsCL GRADIENT ULTRACENTRIFUGATION AND IDENTIFICATION OF ${ }^{13}$ C-ENRICHED DNA}

To separate ${ }^{13} \mathrm{C}$-enriched and unenriched DNA, total extracted DNA from each sample was added to cesium chloride $(\mathrm{CsCl})$ solutions $\left(1.72 \mathrm{~g} \mathrm{ml}^{-1}\right)$ for isopycnic ultracentrifugation and gradient fractionation, as previously described (Jones et al., 2011). As an internal standard for unlabeled DNA, $5 \mu$ l of purified Escherichia coli DNA (ca. $40 \mathrm{ng} \mathrm{ml}^{-1}$ ) was added and mixed into each tube prior to ultracentrifugation. Each fraction was then analyzed by denaturing gradient gel electrophoresis (DGGE) to visualize the separation of DNA. For this, PCR amplification of each fraction was carried out with primers 63f-GC (Marchesi et al., 1998) and 517r (Muyzer et al., 1993) using a PCR program as described by Yu and Morrison (2004). PCR 
products were confirmed on a $1.5 \%(\mathrm{w} / \mathrm{v})$ agarose gel alongside a HindIII DNA ladder (Invitrogen, Carlsbad, CA, USA). DGGE was performed using 6.5\% acrylamide gels containing a denaturant range of 30-60\%. After electrophoresis for $16 \mathrm{~h}$ at $60^{\circ} \mathrm{C}$ and $60 \mathrm{~V}$, gels were stained with ethidium bromide (1:25,000 dilution; $15 \mathrm{~min}$ ). Gel images were captured and visualized using the GNU Image Manipulation Program (GIMP; version 2.6.8).

\section{S rRNA GENE LIBRARIES OF ${ }^{13} \mathrm{C}$-ENRICHED DNA}

$16 \mathrm{~S}$ rRNA clone libraries, each comprising 96 clones, were prepared from combined fractions containing the ${ }^{13} \mathrm{C}$-enriched DNA from each of the duplicate SIP incubations. For this, the general eubacterial primers $27 \mathrm{f}$ and $1492 \mathrm{r}$ were used for amplification of the 16S rRNA gene and then partially sequenced using primer 27f (Wilmotte et al., 1993) at the Beckman Coulter Genomics sequencing facility (Danvers, MA, USA). The ${ }^{13} \mathrm{C}$-enriched heavy DNA fractions were selected based on the DGGE evidence, as discussed below. After excluding vector sequences, poor-quality reads and chimeras, clone sequences were grouped into operational taxonomic units (OTUs) based on applying a 97\% sequence identity cutoff. The complete linkage clustering and dereplicate tools available at the Pyrosequencing Pipeline tool of RDP-II (Cole et al., 2009) were used to select representative sequences for dominant OTUs identified in each of the libraries. Near-complete $16 \mathrm{~S}$ rRNA gene sequences for the represented sequences were obtained at the University of North Carolina-Chapel Hill Genome Analysis Facility. Sequencher 4.8 (Gene Codes Corp., Ann Arbor, MI, USA) was used to edit and assemble these sequences, and the BLASTn search program and RDP-II (Maidak et al., 1999) were used to check for close relatives and phylogenetic affiliation.

\section{REAL-TIME QUANTITATIVE PCR}

To quantify sequences in the dominant OTUs, primers for realtime quantitative PCR (qPCR) were developed using the Probe Design and Probe Match tools of ARB, as previously described (Gutierrez et al., 2011). The Probe Check tool of RDP-II was used to confirm primer specificity, and the optimal annealing temperature of each primer pair was determined using an Eppendorf (Hauppauge, NY, USA) or Applied Biosystems (Foster City, CA. USA) Mastercycler gradient thermal cycler. The template for these reactions, and for the construction of respective standard curves for qPCR, was either a plasmid containing a representative sequence that had been linearized using PstI (New England BioLabs, Ipswich, MA, USA), or a PCR amplicon of the 16S rRNA gene, and purified using the QIAquick nucleotide removal kit (Qiagen, Valencia, CA, USA). The primer pairs, their optimal annealing temperature, amplification efficiency (Pfaffl, 2001), detection limit and RDP hits are shown in Table 1. To confirm the fractions from the DGGE profiles that corresponded to unlabeled DNA, E. coli primers ECP79f (5' -GAAGCTTGCTTCTTTGCT-3') and ECR620r ( $5^{\prime}$-GAGCCCGGGGATTTCACA-3') were used to quantify the abundance of the E. coli $16 \mathrm{~S}$ rRNA genes in each fraction. An annealing temperature of $55^{\circ} \mathrm{C}$ was used for the qPCR program employing these primers (Sabat et al., 2000).
Purified DNA from time-series incubations with unlabeled hydrocarbon was quantified using a NanoDrop ND-3300 fluorospectrometer (Thermo, Waltham, MA, USA) and the Quant-iT Picogreen double-stranded DNA (dsDNA) kit (Invitrogen). As duplicates of the separated ${ }^{12} \mathrm{C}$ - and ${ }^{13} \mathrm{C}$-labeled incubations for each of the three SIP incubations displayed similar distributions of DNA in the fractions, as well as similar DGGE profiles, only the replicate incubation whose fractions contained the highest total amount of DNA was used for further analyses. SIP-identified sequences were quantified in each separated SIP fraction using at least duplicate reactions by $\mathrm{qPCR}$, as described previously (Singleton et al., 2006). Single reactions were performed on each triplicate DNA extraction (from triplicate samples) from the time series containing unlabeled hydrocarbon.

\section{ISOLATION AND DEGRADATION EXPERIMENTS}

The detection of Methylophaga sequences in the heavy DNA clone library from SIP prompted us to isolate these organisms in order to further verify their potential to degrade hydrocarbons. For this, a fresh inoculum was prepared (as described above) from the same batch of North Carolina surface seawater and used to inoculate three 250-ml Erlenmeyer flasks, each containing $50 \mathrm{ml}$ of ONR7a medium amended with methanol supplied via the vapor phase, as previously described (Paje et al., 1997). Methanol, as the sole carbon and energy source, was used in order to preferentially enrich for methylotrophs. After 1-week incubation $\left(250 \mathrm{rpm} ; 21^{\circ} \mathrm{C}\right)$ in the dark, samples $(50 \mu \mathrm{l})$ from each flask were streaked directly onto ONR7a agar plates and stored in a desiccator containing a small beaker containing ca. $50 \mathrm{ml}$ of methanol. Colonies displaying distinct colonial morphologies were picked and subcultured onto fresh ONR7a agar medium (amended with methanol via the vapor phase) until pure cultures were obtained and stored in glycerol $(30 \% \mathrm{v} / \mathrm{v})$ at $-80^{\circ} \mathrm{C}$. One isolate, designated strain SM14, was selected for further study.

The potential of strain SM14 to grow on $n$-hexadecane as the sole carbon and energy source was determined in acid-washed (0.1 N HCl) 250-ml autoclaved glass screw-top Schott bottles with caps lined with aluminium foil to prevent the adsorption of hydrocarbons. Each bottle contained $50 \mathrm{ml}$ of ONR7a medium and $1 \mathrm{mg}$ of unlabeled $n$-hexadecane ( $>99 \%$ purity). One set of triplicate bottles was inoculated with strain SM14 cells that had been washed several times with sterile ONR7a broth. Uninoculated controls, acid-killed controls and bottles that were inoculated, but without any added $n$-hexadecane, were also prepared. All incubations were conducted in triplicate and incubated in the dark with shaking $(150 \mathrm{rpm})$ at $25^{\circ} \mathrm{C}$. Growth was monitored spectrophotometrically by taking triplicate measurements of the culture medium periodically at an optical density of $600 \mathrm{~nm}$. Concentrations of $n$-hexadecane were measured by gas chromatography (GC). For this, triplicate samples $(1 \mathrm{ml})$ were taken at each time point and extracted with $2 \mathrm{ml}$ of ethyl acetate (EA). Heptanone, as internal standard, was added to each of the non-aqueous EA extracts prior to injection $(3 \mu \mathrm{l})$ into a Hewlett Packard 5890 series II GC equipped with an Equity 1 (Supelco) column $(60 \mathrm{~m} \times 0.32 \mathrm{~mm}$ i.d. $)$ and a flame ionization detector. The operating conditions were as follows: column temperature initially $60^{\circ} \mathrm{C}$ for $1 \mathrm{~min}$, then $60-250^{\circ} \mathrm{C}$ at $18^{\circ} \mathrm{C} / \mathrm{min}$, followed 
Table 1 | Quantitative PCR primers developed and used in this study.

\begin{tabular}{|c|c|c|c|c|c|c|c|c|}
\hline Target OTU & Primer name & Primer sequence $\left(5^{\prime} \rightarrow 3^{\prime}\right)$ & $\mathrm{T}_{\mathrm{M}}\left({ }^{\circ} \mathrm{C}\right)^{\mathrm{a}}$ & qPCR standard ${ }^{b}$ & Amplicon length & Amp. Eff. ${ }^{c}$ & Detect. limit ${ }^{d}$ & RDP hits ${ }^{e}$ \\
\hline 1 & Alc-604r & CTGCACTCTAGCYTGCCA & & & & & & $448(6)$ \\
\hline 4 & Met-214r & GGTTCATCTGTCAGCGTGAG & & & & & & $96(83)$ \\
\hline
\end{tabular}

${ }^{a}$ Empirically determined PCR annealing temperature.

${ }^{b}$ Representative clone sequences used to generate standard curves. Names are as in Figures 3, 5.

${ }^{c}$ Amp. Eff., amplification efficiency (Pfaffl, 2001) with OTU-specific primers.

${ }^{d}$ Detection limit of each qPCR assay expressed as number of $16 S$ rRNA gene copies per milliliter of culture.

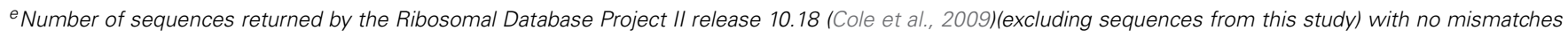
to primer pairs. Values in parentheses are the total hits that each pair of primers target.

by holding at $250^{\circ} \mathrm{C}$ for $18.5 \mathrm{~min}$; injector temperature at $300^{\circ} \mathrm{C}$; detector temperature at $310^{\circ} \mathrm{C}$; and carrier gas $\mathrm{He}\left(1.0 \mathrm{ml} \mathrm{min}^{-1}\right)$.

\section{PHYLOGENETIC TREE}

The 16S rRNA sequences of the isolated strain and SIP-identified representative sequences were aligned using CLUSTAL_X (Thompson et al., 1994) with close relatives as determined by RDP and BLASTn searches of GenBank. A neighbor-joining tree was constructed with bootstrapped replication (1000 times) and Escherichia coli 0157:H7 (AY513502) was used as an outgroup.

\section{NUCLEOTIDE SEOUENCE ACCESSION NUMBERS}

The following accession numbers were submitted to GenBank for ${ }^{13} \mathrm{C}$-enriched DNA from SIP experiment with $n$-hexadecane: Alcanivorax sp. clone HEX19 (KF875698) and Methylophaga sp. clone HEX76 (KF790924). The accession number for Methylophaga sp. strain SM14 is KF790925.

\section{RESULTS}

\section{INCUBATIONS WITH LABELED AND UNLABELED n-HEXADECANE}

During the SIP experiment, incubations containing unlabeled or ${ }^{14} \mathrm{C}$-labeled $n$-hexadecane were run in parallel to measure, respectively, for the disappearance and mineralization of this hydrocarbon. As shown in Figure 1, complete removal of $n$-hexadecane occurred by day 5 , whereas mineralization of ${ }^{14} \mathrm{C}$ associated with $n$-hexadecane continued up until day 7 . The endpoint selected for the extraction of DNA from the ${ }^{13} \mathrm{C}$ incubations was 7 days, which corresponded to slowing of the mineralization rate for the $n$-hexadecane. The DNA extracts (from each of the duplicate ${ }^{13} \mathrm{C}$ incubations) was subjected to isopycnic ultracentrifugation to isolate the ${ }^{13} \mathrm{C}$-enriched "heavy" DNA for subsequent analysis.

\section{DNA GRADIENT ULTRACENTRIFUGATION AND IDENTIFICATION OF LABELED 16S rRNA GENES}

DGGE analysis of the fractions from the SIP incubations showed clear evidence of isotopic enrichment of DNA in $\left[{ }^{13} \mathrm{C}\right] n$ hexadecane incubations, separation of ${ }^{13} \mathrm{C}$-labeled and unlabeled DNA, and different banding patterns between the ${ }^{13} \mathrm{C}$-enriched and unenriched DNA fractions (Figure 2). For the ${ }^{13} \mathrm{C}$ incubations shown in Figure 2, fractions 7-10 were combined and used to construct the $16 \mathrm{~S}$ rRNA gene clone library. Fractions from the duplicate gradient (i.e., of the duplicate ${ }^{13} \mathrm{C}$ incubation) were

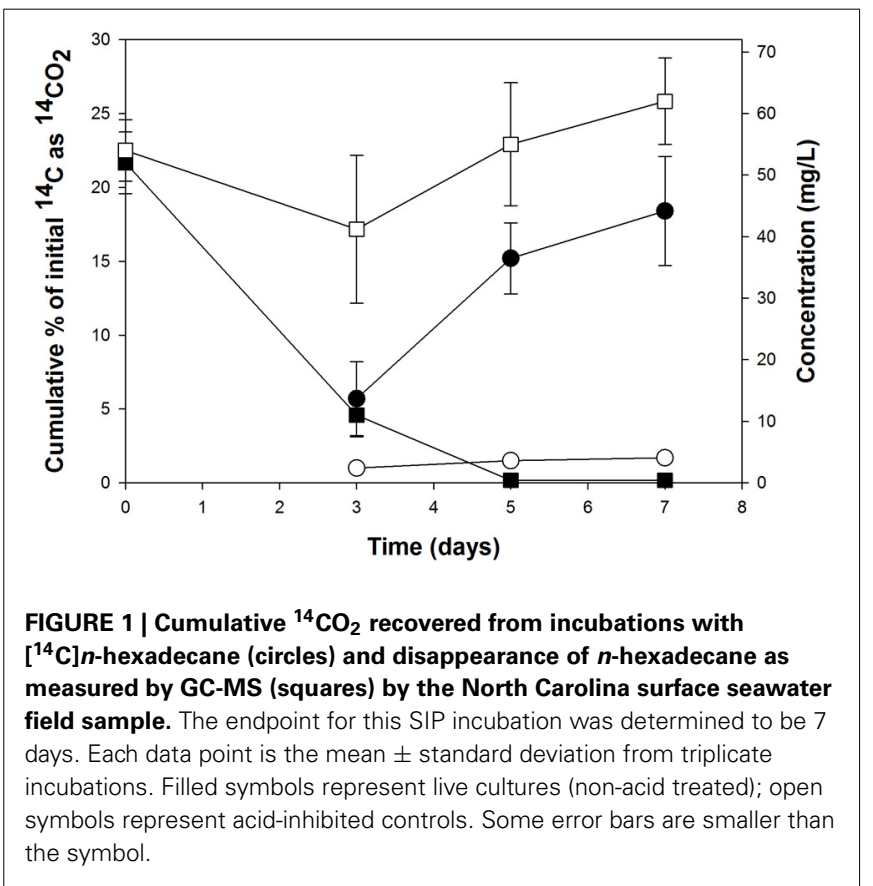

combined and similarly manipulated (data not shown). After excluding vector sequences, poor sequence reads, chimeras, and singleton sequences, the clone library constructed from pooled ${ }^{13} \mathrm{C}$-enriched DNA comprised 85 sequences. Table 2 shows the OTU representation in the clone library together with the phylogenetic affiliation based on a BLASTn search in GenBank. Of the 85 sequences, 5 OTUs were identified based on a $>97 \%$ sequence identity cutoff. OTU-1 (71 sequences) comprised the majority $(84 \%)$ of the 85 sequences and was found affiliated to the genus Alcanivorax. OTU-2 (4 sequences) and OTU-5 (4 sequences) were affiliated to the genus Marinobacter and shared 95\% sequence identity. OTU-3 (3 sequences) and OTU-4 (3 sequences) were, respectively, affiliated to Oleibacter and Methylophaga. All other OTUs in the clone library were represented by single sequences and are presented in Supplementary Table 1.

Primers for qPCR were designed targeting the 16S rRNA gene of the most dominant SIP-identified group, Alcanivorax OTU-1 (Table 1), in order to quantify this group in the "heavy" DNA 
from the SIP incubations (Figure 2) and in incubations with unlabeled $n$-hexadecane to confirm its enrichment (Figure 3). As shown in Figure 2, qPCR detection of this group was largely confined to the "heavy" DNA (fractions 7-10), thus confirming its enrichment of the ${ }^{13} \mathrm{C}$ label from $\left[{ }^{13} \mathrm{C}\right] n$-hexadecane. By day 5 in the unlabeled incubations (Figure 3), the gene copy number of this group increased by ca. 5 orders of magnitude, coinciding with the disappearance and mineralization of the $n$-hexadecane (Figure 1). This increase in gene copy number also coincided with

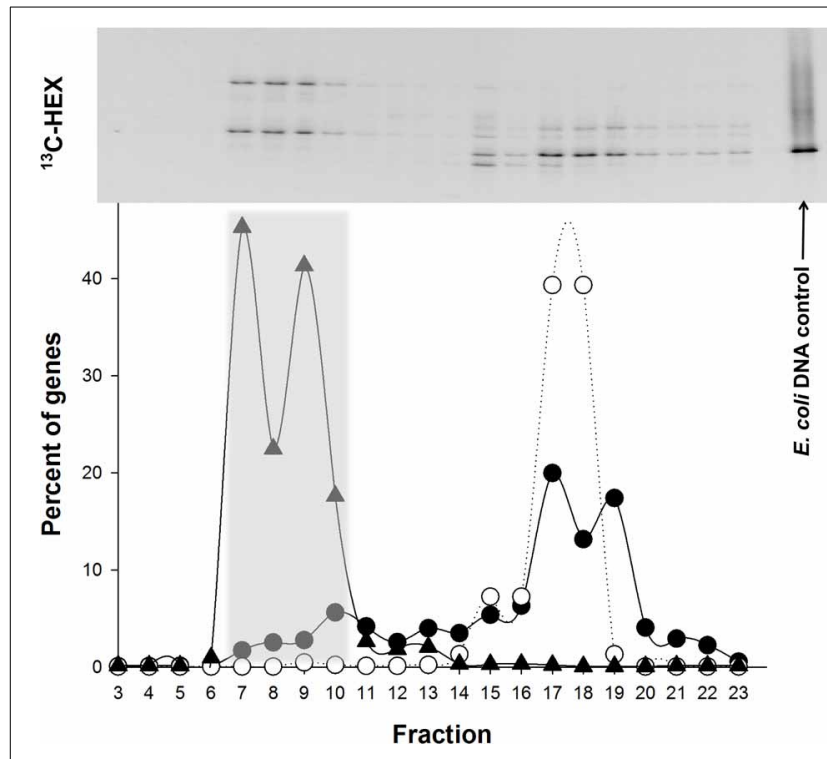

FIGURE 2 | Distribution of the "heavy" and "light" DNA in separated SIP fractions. (Top) DGGE image of bacterial PCR products from separated $\left[{ }^{13} \mathrm{C}\right] n$-hexadecane fractions, with decreasing densities from left to right. The position of unlabeled E. coli DNA, which was used as an internal control in the isopycnic centrifugation, is shown on the right. (Bottom) Distribution of qPCR-quantified 16S rRNA gene sequences is shown below the DGGE image for Alcanivorax (triangles) and Methylophaga (solid circles) in fractions from the $\left[{ }^{13} \mathrm{C}\right] n$-hexadecane incubations. The distribution of qPCR-quantified 16S rRNA gene sequences for $E$. coli is also shown (open circles) in fractions from the ${ }^{13} \mathrm{C}$ incubation. Gene copies in a fraction are presented as a percentage of the total genes quantified in the displayed range of fractions. Data points are aligned with equivalent fractions of the DGGE image. an increase in the total concentration of DNA as indicator of cell growth. The observed significant increase in the 16S rRNA gene copy number of this organism by day 5 coupled with its growth (total DNA as proxy), the disappearance and mineralization of the $n$-hexadecane, and appearance of respective $16 \mathrm{~S}$ rRNA genes in only the most heavily ${ }^{13} \mathrm{C}$-enriched DNA fractions (Figure 2) of incubations containing the ${ }^{13} \mathrm{C}$-labeled substrate, strongly supports the enrichment of this Alcanivorax on the $n$-hexadecane as a growth substrate.

A similar approach was used to confirm the apparent enrichment of Methylophaga OTU-4 in the clone library constructed from ${ }^{13} \mathrm{C}$-enriched DNA (Table 2) and link this organism to the degradation of $n$-hexadecane. As shown in Figure 2, 13\% of total Methylophaga genes were confined to the "heavy" DNA (fractions 7-10) compared to $56 \%$ in the "light" DNA (fractions 16-19), thus indicating partial enrichment of this organism in the ${ }^{13} \mathrm{C}$-enriched DNA. Figure 3 shows that the gene copy number of this group increased by ca. 3 orders of magnitude by day 3 ,

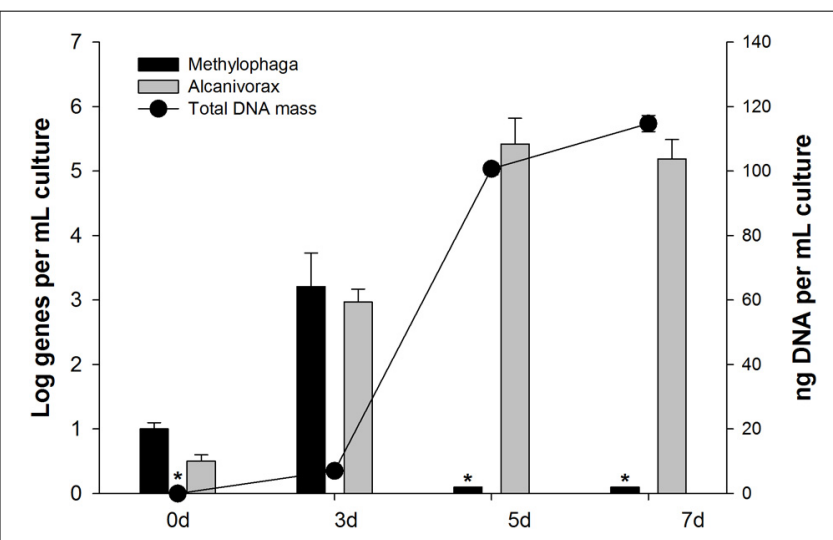

FIGURE 3 | Abundance of Alcanivorax and Methylophaga 16S rRNA genes during incubation with unlabeled $\boldsymbol{n}$-hexadecane. Bars are the mean \pm standard deviations of results from triplicate $\mathrm{gPCR}$ measuring the abundance of group-specific $16 \mathrm{~S}$ rRNA genes. Circles are the mean \pm standard deviations of triplicate measurements of the total mass of DNA per sample. Bars or data points with asterisks represent numbers with one or more readings below the quantification limit of the assay and are presented as the largest possible value for that point.

Table 2 | SIP-identified sequences in the clone library constructed from ${ }^{13} \mathrm{C}$-enriched DNA ${ }^{\mathrm{a}}$.

\begin{tabular}{|c|c|c|c|c|}
\hline OUT no. & Rep. seq. ${ }^{b}$ & Closest BLASTn match ${ }^{c}$ & Accessioin no. & Clones in library $(\%)^{d}$ \\
\hline 1 & HEX19 & Alcanivorax jadensis (98\%) & AJ001150 & 71 \\
\hline 2 & HEX85 & Marinobacter lipolyticus (97\%) & AY147906 & 4 \\
\hline 3 & HEX49 & Oleibacter marinus (99\%) & AB435650 & 3 \\
\hline 4 & HEX76 & Methylophaga thiooxydans (97\%) & DQ660915 & 3 \\
\hline 5 & HEX17 & Marinobacter flavimaris (100\%) & AY517632 & 4 \\
\hline
\end{tabular}

${ }^{a}$ HEX, SIP with [U- ${ }^{13} \mathrm{C}$ ]n-hexadecane.

${ }^{b}$ Representative sequence for each OTU. Singleton sequences are listed in Supplementary Table 1.

${ }^{c}$ Results are to the closest type strain; percentage similarity shown in parentheses.

d Total number of sequences in ${ }^{13} \mathrm{C}$-enriched DNA clone library from the $n$-hexadecane SIP incubation was 85 . A $97 \%$ cut-off was used to classify sequences to an OTU. 
which coincided with ca. $80 \%$ disappearance of the $n$-hexadecane, $6 \%$ of ${ }^{14} \mathrm{C}$ mineralized (as ${ }^{14} \mathrm{CO}_{2}$ ) of initial ${ }^{14} \mathrm{C}$ (as ${ }^{14} \mathrm{C}$-labeled $n$-hexadecane) and increase in total DNA concentration (as indicator of growth). At days 5 and 7, the gene copy number of this organism declined to reflect initial numbers $(<0.5$ Log genes per $\mathrm{mL}$ of culture).

\section{GROWTH ON AND DEGRADATION OF n-HEXADECANE BY METHYLOPHAGA SP. STRAIN SM14}

Enrichment experiments using the North Carolina field sample as inoculum and with methanol as the sole carbon and energy source yielded two isolates, designated strain SM13 and strain SM14. Both strains were found affiliated to the genus Methylophaga based on sequencing of their 16S rRNA genethey shared $94 \%$ sequence identity between them. Strain SM13 proved difficult to maintain in laboratory culture as it eventually ceased to grow upon subsequent subculturing and was therefore no longer used for further experimentation. On the other hand, strain SM14 yielded small $(0.05-0.15 \mathrm{~mm})$ off-white colonies after 2 weeks incubation on ONR7a agar amended with Na-pyruvate as the sole carbon and energy source (not shown). The strain also grew well in ONR7a broth amended with Na-pyruvate or methanol. Evidence of the strain's ability to grow on $n$-hexadecane as a sole carbon and energy source is shown in Figure 4. At an initial $n$-hexadecane concentration of $0.002 \%(\mathrm{v} / \mathrm{v})$, the strain reached a low cell density of ca. 0.015 at $600 \mathrm{~nm}$. However, growth coincided with the disappearance of the $n$-hexadecane, which was indicative that the hydrocarbon was being degraded by the strain and utilized as a carbon source for growth. No growth was measured in uninoculated controls, or in inoculated incubations in the absence of any added $n$-hexadecane. Cultures amended with higher concentrations of $n$-hexadecane yielded higher cell densities (results not shown). Evidence that this was a pure culture of Methylophaga strain SM14 was confirmed by 16S rRNA gene sequencing of DNA isolated from cell pellets collected toward the end of these growth experiments.

\section{PHYLOGENETIC ANALYSIS}

The near-complete 16S rRNA gene sequence of the major SIPidentified OTUs and Methylophaga strain SM14 were used to construct a phylogenetic tree with related sequences from GenBank (Figure 5). The representative sequence for Alcanivorax identified from incubations with ${ }^{13} \mathrm{C}$-hexadecane (HEX19; OTU1) shared highest $16 \mathrm{~S}$ rRNA sequence identity (98\%) with Alcanivorax jadensis $\mathrm{T}^{\mathrm{T}}$ (Fernandez-Martinez et al., 2003)previously (Fundibacter) jadensis that had been isolated from intertidal sediment collected from the German North Sea coast (Bruns and Berthe-Corti, 1999); second highest sequence identity (97\%) was with Alcanivorax borkumensis SK2 $2^{\mathrm{T}}$ that was isolated from seawater/sediment samples collected near the Isle of Borkum in the North Sea (Yakimov et al., 1998).

The 16S rRNA sequence of Methylophaga strain SM14 shared 99.9\% identity with SIP clone HEX76 (Methylophaga OTU-4). Highest sequence identity for both SM14 and clone HEX76 was found with two uncultured Methylophaga clones, DOM03 and DOM14 (97.5\% similarity), identified associated with marine dissolved organic matter (DOM) (McCarren et al., 2010). Highest

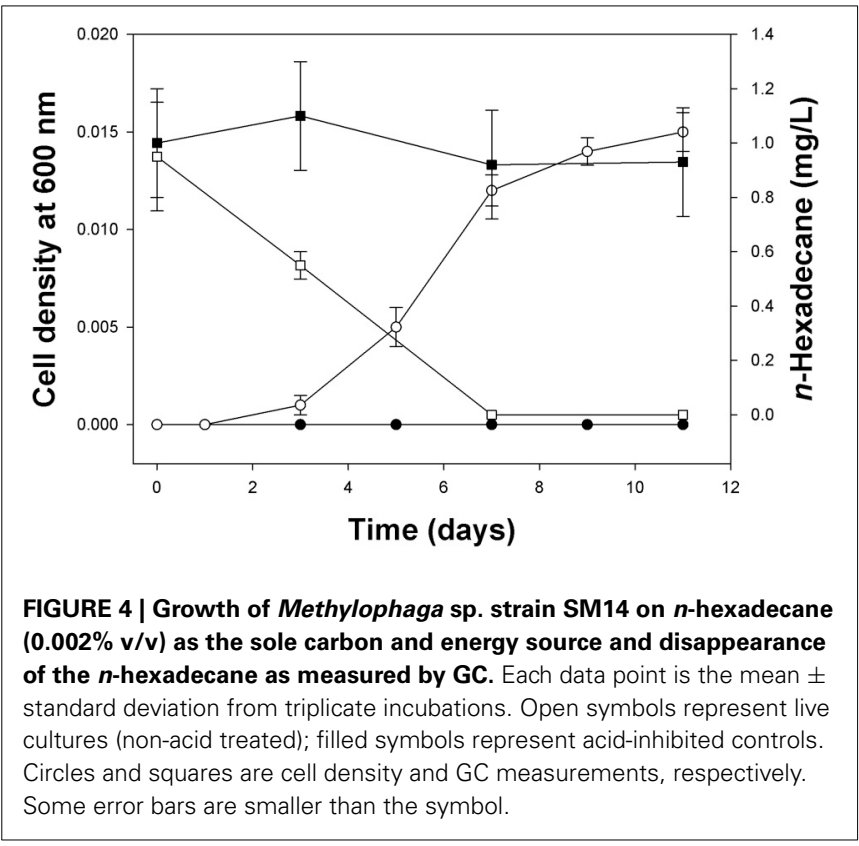

sequence identity of SM14 and HEX76 to type strains was with M. thiooxydans DMS010 ${ }^{\mathrm{T}}$ (97.1\%) isolated from an enrichment culture of the coccolithophorid Emiliana huxleyi with dimethylsulfide (Schäfer, 2007; Boden et al., 2010), whereas they shared $\leq 95 \%$ to other Methylophaga type strains. To further clarify the phylogenetic identity of strain SM14, tree construction was also performed with the neighbor-joining, maximum parsimony, and maximum likelihood methods. In all cases, the position of strain SM14 always grouped to the genus Methylophaga.

\section{DISCUSSION}

SIP has proven useful for linking the phylogenetic identity of microorganisms with a metabolic function. However, careful attention must be employed in its design and execution in order for it to yield interpretable, unambiguous results. One of the main challenges in SIP is obtaining sufficient incorporation of the ${ }^{13} \mathrm{C}$ into biomass, which in the case for DNA-SIP, its enrichment into DNA. Whilst the extent of labeling can be increased with longer incubation times, this can lead to the ${ }^{13} \mathrm{C}$ becoming distributed among other members of the microbial communityi.e., that are not necessarily directly capable of metabolizing the isotopically-labeled substrate-by cross-feeding on ${ }^{13} \mathrm{C}$-labeled metabolic byproducts, intermediates, or dead cells (Leuders et al., 2004). To avert this, we had set up several ${ }^{12} \mathrm{C}$ and ${ }^{14} \mathrm{C}$ incubations that ran in parallel to the ${ }^{13} \mathrm{C}$ incubations in order to tractably measure for the degradation (by GC-MS) and mineralization (by scintillation counts) of the $n$-hexadecane to help guide our selection of the point at which to terminate the ${ }^{13} \mathrm{C}$ incubations (endpoint of experiment) whereby sufficient ${ }^{13} \mathrm{C}$ incorporation had been achieved with minimal cross-feeding. The absence of a heavy DNA band in the ${ }^{12} \mathrm{C}$ controls (not shown) and the distinct bimodal distribution between ${ }^{13} \mathrm{C}$ and ${ }^{12} \mathrm{C}$ DNA bands on DGGE confirmed the incorporation of the label from $\left[{ }^{13} \mathrm{C}\right] n$-hexadecane by a subgroup of the total microbial community. 


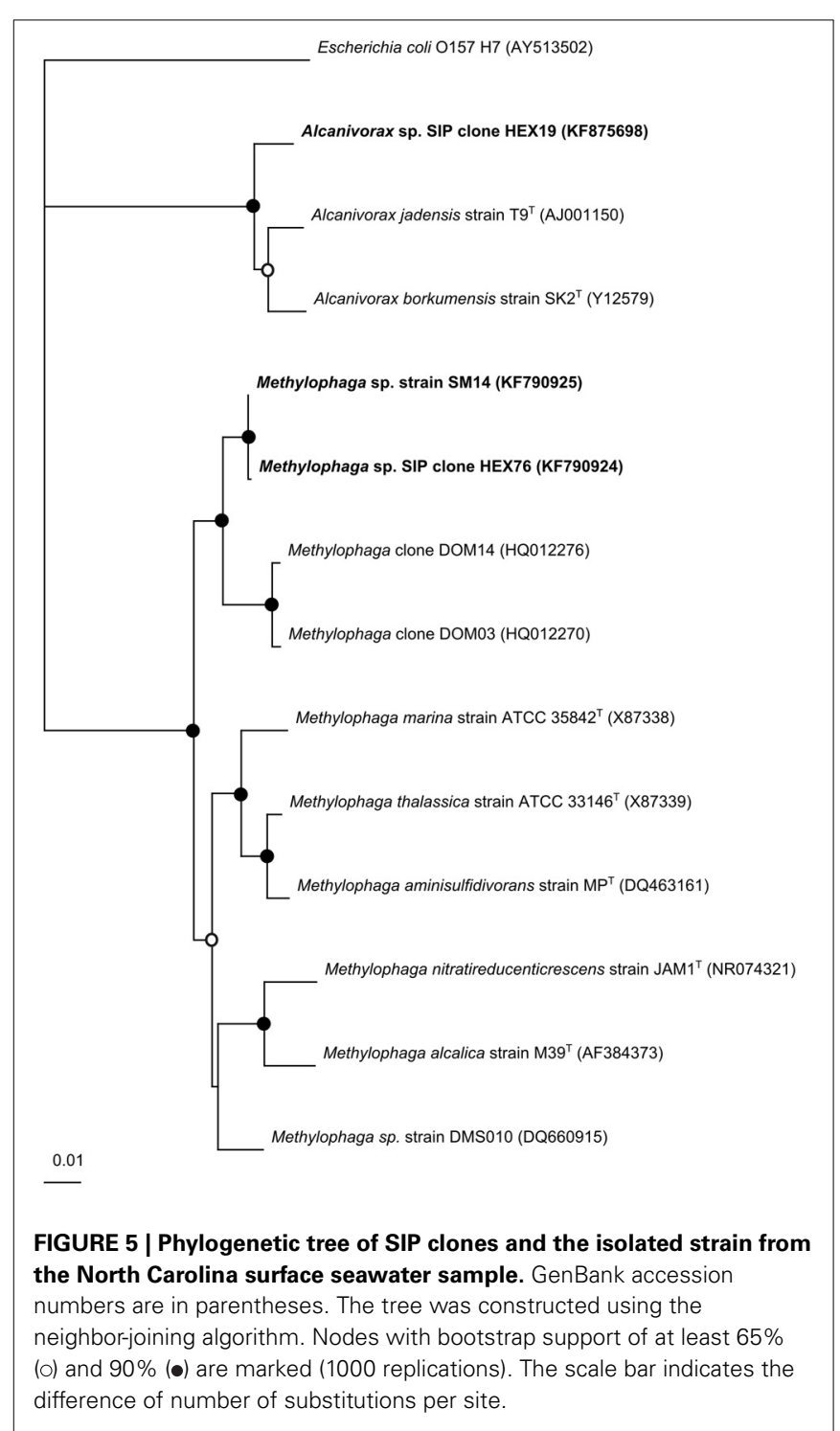

Based on the clone libraries constructed from ${ }^{13} \mathrm{C}$-enriched DNA, the main consumers of the $\left[{ }^{13} \mathrm{C}\right] n$-hexadecane were affiliated with Alcanivorax — a group of cosmopolitan bacteria that utilize petroleum oil hydrocarbons almost exclusively as a preferred carbon and energy source. Since this SIP-identified Alcanivorax, represented by OTU-1 (clone HEX19), was found with $\geq 2 \% 16 \mathrm{~S}$ rRNA sequence difference to closely related type strains, it is likely to represent a new phylogenetic taxon within this genus. Further confirmation for the enrichment of this Alcanivorax OTU and its dominant role in the degradation of the $n$-hexadecane was provided by $\mathrm{qPCR}$ which revealed a dramatic increase in the abundance of the 16S rRNA gene copy number for these organisms in the unlabeled incubations (Figure 3). In addition, since growth of these organisms coincided with disappearance of the $n$-hexadecane, and appearance of their 16S rRNA genes in only the most heavily enriched ${ }^{13} \mathrm{C}$-DNA fractions, suggests that their presence in the clone libraries constructed from heavy DNA was unlikely due to cross-feeding. Other possible contributors to the consumption of the $\left[{ }^{13} \mathrm{C}\right] n$-hexadecane during SIP were Marinobacter and Oleibacter, as several sequences (3-9\% of the total clone library) were found affiliated with these genera in the libraries constructed from heavy DNA-members of these genera are commonly found enriched during oil spills in marine waters and can play a major role in the degradation of aliphatic hydrocarbons, such as $n$-hexadecane.

Intriguingly, several sequences affiliated to Methylophaga were also identified in the heavy DNA clone libraries (ca. $4 \%$ of the total clone library), and since this SIP-identified Methylophaga (represented by OTU-4) was found with $\geq 3 \%$ 16S rRNA sequence difference to closely related type strains, it is likely to represent a new phylogenetic taxon within this genus. To our knowledge, this represents the first identification of Methylophaga by SIP targeting hydrocarbon degraders using a ${ }^{13} \mathrm{C}$-labeled hydrocarbon. Based on the presence of these organisms in the heavy DNA fractions (Figure 2) and increased abundance of their 16S rRNA gene copy number by day 3 in the unlabeled incubations - which coincided with the disappearance and mineralization of the $n$-hexadecane-these results supported the contribution of the respective newly identified Methylophaga (OTU-4) to the degradation of the hydrocarbon. We are unable at the present time to explain why the initial increase in their gene copy number was, by day 5 , subsequently followed by a regression in their abundance to below detection limits. We observed similar results during a crude oil enrichment experiment in which pyrosequencing was used to analyse the bacterial community response associated with the marine diatom Skeletonema costatum to reveal an initial and distinctive bloom in Methylophaga (unpublished results). As the SIP experiment was designed to minimize the possibility of cross-feeding, and the various analyses performed (i.e., DNA quantification, qPCR of target genes, etc) support the enrichment of Methylophaga on the $n$-hexadecane as a growth substrate within at least the first 3 days of the experiment, it is highly unlikely that any Methylophaga DNA had migrated into the heavy fractions during isopycnic ultracentrifugation unless it was enriched with ${ }^{13} \mathrm{C}$. This is further supported by the fact that no single sequence of the internal standard (unlabeled E. coli DNA) was detected in the heavy fractions and clone libraries constructed from the heavy DNA. It is more likely, in fact, that Methylophaga sequences were underrepresented in ${ }^{13} \mathrm{C}$-enriched DNA clone libraries because the SIP experiment was terminated (day 7) after the apparent peak in growth of Methylophaga occurred (day 3; Figure 3).

In order to further validate whether the SIP-identified Methylophaga (OTU-4) were capable of utilizing $n$-hexadecane as a sole carbon and energy source, we isolated a member of this genus, Methylophaga strain SM14, that shared 99.9\% 16S rRNA gene sequence identity to the representative sequence for this OTU (i.e., SIP clone HEX76) and directly demonstrated its ability to grow on and degrade $n$-hexadecane as a sole carbon and energy source (Figure 4). Collectively, our results present compelling evidence to implicate, for this first time, a novel member of the genus Methylophaga in hydrocarbon degradation, hence expanding the substrate spectrum for certain members of this genus beyond solely utilizing $\mathrm{Cl}$ carbon sources-with the exception of a few species able to also utilize fructose. 
The discovery of hydrocarbon-degrading Methylophaga has important implications to assessing their role in the fate of the oil that entered the Gulf of Mexico during the Deepwater Horizon spill. During the active phase of the spill (April 20 to July 15), methylotrophic bacteria (incl. Methylophaga) were not detected near the leaky wellhead (Hazen et al., 2010; Valentine et al., 2010; Yang et al., 2014), whereas these organisms were reported to account for at least $5 \%$ of the total bacterial community after the spill-i.e., Kessler et al. (2011) reported 5-36\% in Sep. 2010; Yang et al. (2014) report 0.23-6\% and 2\% in Sep. 2010 and Oct. 2010, respectively. However, a recent report by Dubinsky et al. (2013) which analyzed the microbial community composition of plume waters during June and August 2010 - a time period not covered in these earlier reports-describes the start of a Methylophaga enrichment in plume waters during late June that appeared to be sustained until late August. Furthermore, transcriptional analysis by Rivers et al. (2013) of water column samples collected during the active phase of the spill (May 26 to June 3, 2010) revealed that Methylophaga were in a heightened state of metabolic activity within the plume relative to non-plume waters. Kessler et al. (2011) postulated that methylotrophs, such as Methylophaga, had contributed to the consumption of the methane released during the spill. However, such organisms are not recognized for carrying out the oxidation, or being capable of growing on, methane as a carbon and energy source. Hence, their potential to have consumed the methane remains contentious in the absence of substantive evidence to support this. Our results from SIP and with strain SM14 herein provide evidence to reassess the possibility that a subset of the Methylophaga community in the Gulf of Mexico, specifically those with hydrocarbon-degrading potential, may have contributed to the degradation of some components of the oil (e.g., saturated hydrocarbons). Dubinsky et al. (2013) measured higher-than-background levels of BTEX, cyclo-alkanes and $n$-alkanes in plume waters during the Methylophaga-enrichment phase in late June that, hence, might have acted as potential carbon and energy sources for these organisms. We hypothesize that the apparent enrichment of these organisms, albeit ephemerally, during the latter phase of the spill may have been in part associated with the potential for some members of this genus to degrade hydrocarbons.

\section{AUTHOR CONTRIBUTIONS}

Sara Mishamandani, Tony Gutierrez, and Michael D. Aitken contributed to the design of the work and its interpretation. Sara Mishamandani and Tony Gutierrez produced all of the data and, together with Michael D. Aitken, drafted and critically revised the manuscript and approved the final version for publication.

\section{ACKNOWLEDGMENTS}

We thank Frank Schwartz for providing us the opportunity to take samples during a field trip offshore the NC coast, and Janelle Fleming for additional assistance with our sampling needs. This work was supported by a Marie Curie International Outgoing Fellowship (PIOF-GA-2008-220129) within the 7th European Community Framework Programme. Partial support was also provided through the US National Institute of Environmental Health Sciences, grant 5 P42ES005948. Sara Mishamandani was supported by a Summer Undergraduate Research Fellowship from the Office for Undergraduate Research at the University of North Carolina at Chapel Hill and by the Kimball King Undergraduate Research Fund administered by the UNC Honors Office. We also thank Scott Hauswirth for assistance with the GC-MS analysis at UNC Chapel Hill, James MacKinlay for assistance with the GC analysis at Heriot-Watt, David Singleton for assistance with qPCR, and Kirk Semple for providing $\left[1-{ }^{14} \mathrm{C}\right] n$ hexadecane. Finally, we would like to also thank the two reviewers for their valuable comments during the preparation of the manuscript.

\section{SUPPLEMENTARY MATERIAL}

The Supplementary Material for this article can be found online at: http://www.frontiersin.org/journal/10.3389/fmicb. 2014.00076/abstract

Supplementary Table 1 | Singleton 16S rRNA gene sequences recovered from the heavy DNA clone library ${ }^{a}$.

\section{REFERENCES}

Boden, R., Kelly, D. P., Murrell, J. C., and Schäfer, H. (2010). Oxidation of dimethylsulfide to tetrathionate by Methylophaga thiooxidans sp. nov.: a new link in the sulphur cycle. Environ. Microbiol. 12, 2688-2699. doi: 10.1111/j.14622920.2010.02238.x

Bruns, A., and Berthe-Corti, L. (1999). Fundibacter jadensis gen. nov., sp. nov., a new slightly halophilic bacterium, isolated from intertidal sediment. Int. J. Syst. Bacteriol. 49, 441-448. doi: 10.1099/00207713-49-2-441

Cole, J. R., Wang, Q., Cardenas, E., Fish, J., Chai, B., Farris, R. J. et al. (2009). The ribosomal database project: improved alignments and new tools for rRNA analysis. Nucleic Acids Res. 37, D141-D145. doi: 10.1093/nar/gkn879

Coulon, F., McKew, B. A., Osborn, A. M., McGenity, T. J., and Timmis, K. N. (2007). Effects of temperature and biostimulation on oil-degrading microbial communities in temperate estuarine waters. Environ. Microbiol. 9, 177-186. doi: 10.1111/j.1462-2920.2006.01126.x

Dubinsky, E. A., Conrad, M. E., Chakraborty, R., Bill, M., Borglin, S. E., Hollibaugh, J. T., et al. (2013). Succession of hydrocarbon-degrading bacteria in the aftermath of the Deepwater Horizon oil spill in the Gulf of Mexico. Environ. Sci. Technol. 47, 10860-10867. doi: 10.1021/es401676y

Dumont, M., and Murrell, J. (2005). Stable isotope probing - linking microbial identity to function. Nat. Rev. Microbiol. 3, 499-504. doi: 10.1038/nrmicro1162

Dyksterhouse, S. E., Gray, J. P., Herwig, R. P., Cano Lara, J., and Staley, J. T. (1995). Cycloclasticus pugetii gen. nov., sp. nov., an aromatic hydrocarbon- degrading bacterium from marine sediments. Int. J. Syst. Bacteriol. 45, 116-123. doi: 10.1099/00207713-45-1-116

Fernandez-Martinez, J., Pujalte, M. J., Garcia-Martinez, J., Mata, M., Garay, E., and Rodriguez-Valera, F. (2003). Description of Alcanivorax venustensis sp. nov. and reclassification of Fundibacter jadensis DSM $12178^{T}$ (Bruns and BertheCorti 1999) as Alcanivorax jadensis comb. nov., members of the amended genus Alcanivorax. Int. J. Syst. Evol. Microbiol. 53, 331-338. doi: 10.1099/ijs.0.01923-0

Floodgate, G. D. (1995). Some environmental aspects of marine hydrocarbon bacteriology. Aquat. Microb. Ecol. 9, 3-11. doi: 10.3354/ame009003

Gutierrez, T., Singleton, D. R., Aitken, M. D., and Semple, K. T. (2011). Stableisotope probing of an algal bloom identifies uncultivated members of the Rhodobacteraceae associated with low molecular-weight PAH degradation. Appl. Environ. Microbiol. 77, 7856-7860. doi: 10.1128/AEM.06200-11

Gutierrez, T., Singleton, D. R., Berry, D., Yang, T., Aitken, M. D., and Teske, A. (2013). Hydrocarbon-degrading bacteria enriched by the Deepwater Horizon oil spill identified by cultivation and DNA-SIP. ISME J. 7, 2091-2104. doi: 10.1038/ismej.2013.98

Hazen, T. C., Dubinsky, E. A., DeSantis, T. Z., Andersen, G. L., Piceno, Y. M., Singh, N., et al. (2010). Deep-sea oil plume enriches indigenous oil-degrading bacteria. Science 330, 204-208. doi: 10.1126/science.1195979

Head, I. M., Jones, D. M., and Röling, W. F. (2006). Marine microorganisms make a meal of oil. Nat. Rev. Microbiol. 4, 173-182. doi: 10.1038/nrmicro1348 
Head, I. M., and Swannell, R. P. J. (1999). Bioremediation of petroleum hydrocarbon contaminants in marine habitats. Curr. Opin. Biotech. 10, 234-239. doi: 10.1016/S0958-1669(99)80041-X

Janvier, M., and Grimont, P. A. D. (1995). The genus Methylophaga, a new line of descent within phylogenetic branch $\gamma$ of Proteobacteria. Res. Microbiol. 146, 543-550. doi: 10.1016/0923-2508(96)80560-2

Jones, M. D., Singleton, D. R., Sun, W., and Aitken, M. D. (2011). Multiple DNA extractions coupled with stable-isotope probing of anthracene-degrading bacteria in contaminated soil. Appl. Environ. Microbiol. 77, 2984-2991. doi: 10.1128/AEM.01942-10

Kessler, J. D., Valentine, D. L., Redmond, M. C., Du, M., Chan, E. W., Mendes, S. D. et al. (2011). A persistent oxygen anomaly reveals the fate of spilled methane in the deep Gulf of Mexico. Science 331, 312-315. doi: 10.1126/science.1199697

Leuders, T., Wagner, B., Claus, P., and Friedrich, M. (2004). Stable isotope probing of rRNA and DNA reveals a dynamic methylotroph community and trophic interactions with fungi and protozoa in oxic rice field soil. Environ. Microbiol. 6, 60-72. doi: 10.1046/j.1462-2920.2003.00535.x

Maidak, B. L., Cole, J. R., Parker, C. T. Jr., Garrity, G. M., Larsen, N., Li, B. et al. (1999). A new version of the RDP (Ribosomal Database Project). Nucleic Acids Res. 27, 171-173. doi: 10.1093/nar/27.1.171

Marchesi, J. R., Sato, T., Weightman, A. J., Martin, T. A., Fry, J. C., Hiom, S. J. et al. (1998). Design and evaluation of useful bacterium-specific PCR primers that amplify genes coding for bacterial 16S rRNA. Appl. Environ. Microbiol. 64, 795-799.

McCarren, J., Becker, J. W., Repeta, D. J., Shi, Y., Young, C. R., Malmstrom, R. R., et al. (2010). Microbial community transcriptomes reveal microbes and metabolic pathways associated with dissolved organic matter turnover in the sea. Proc. Natl. Acad. Sci. U.S.A. 107, 16420-16427. doi: $10.1073 /$ pnas. 1010732107

Muyzer, G., de Waal, E. C., and Uitterlinden, A. G. (1993). Profiling of complex microbial populations by denaturing gradient gel electrophoresis analysis of polymerase chain reaction-amplified genes coding for $16 \mathrm{~S}$ rRNA. Appl. Environ. Microbiol. 59, 695-700.

Paje, M. L., Neilan, B., and Couperwhite, I. (1997). A Rhodococcus species that thrives on medium saturated with liquid benzene. Microbiology 143, 2975-2981. doi: 10.1099/00221287-143-9-2975

Pfaffl, M. W. (2001). A new mathematical model for relative quantification in realtime RT-PCR. Nucleic Acids Res. 29, 2002-2007. doi: 10.1093/nar/29.9.e45

Rivers, A. R., Sharma, S., Tringe, S. G., Martin, J., Joye, S. B., and Moran, M. A. (2013). Transcriptional response of bathypelagic marine bacterioplankton to the Deepwater Horizon oil spill. ISME J. 7, 2315-2329. doi: 10.1038/ismej.2013.129

Röling, W. F. M., Milner, M. G., Jones, D. M., Fratepietro, F., Swannell, R. P. J., Daniel, F., et al. (2004). Bacterial community dynamics and hydrocarbon degradation during a field-scale evaluation of bioremediation on a mudflat beach contaminated with buried oil. Appl. Environ. Microbiol. 70, 2603-2613. doi: 10.1128/AEM.70.5.2603-2613.2004

Röling, W. F. M., Milner, M. G., Martin Jones, D., Lee, K., Daniel, F., Swannell, R. J. P., et al. (2002). Robust hydrocarbon degradation and dynamics of bacterial communities during nutrient-enhanced oil spill bioremediation. App. Environ. Microbiol. 68, 5537-5548. doi: 10.1128/AEM.68.11.5537-5548.2002

Sabat, G., Rose, P., Hickey, W. J., and Harkin, J. M. (2000). Selective and sensitive method for PCR amplification of Escherichia coli 16S rRNA genes in soil. Appl. Environ. Microbiol. 66, 844-849. doi: 10.1128/AEM.66.2.844-849.2000

Schäfer, H. (2007). Isolation of Methylophaga spp. from marine dimethylsulfidedegrading enrichment cultures and identification of polypeptyides induced during growth on dimethylsulfide. Appl. Environ. Microbiol. 73, 2580-2591. doi: 10.1128/AEM.02074-06

Singleton, D. R., Sangaiah, R., Gold, A., Ball, L. M., and Aitken, M. D. (2006). Identification and quantification of uncultivated Proteobacteria associated with pyrene degradation in a bioreactor treating PAH-contaminated soil. Environ. Microbiol. 8, 1736-1745. doi: 10.1111/j.1462-2920.2006.01112.x
Teira, E., Lekunberri, I., Gasol, J. M., Nieto-Cid, M., Alvarez-Salgado, X. A., and Figueiras, F. G. (2007). Dynamics of the hydrocarbon-degrading Cycloclasticus bacteria during mesocosm-simulated oil spills. Environ. Microbiol. 9, 2551-2562. doi: 10.1111/j.1462-2920.2007.01373.x

Thompson, J. D., Higgins, D. G., and Gibson, T. J. (1994). CLUSTAL_X: improving the sensitivity of progressive multiple sequence alignment through sequence weighting, position-specific gap penalties and weight matrix choice. Nucleic Acids Res. 22, 4673-4680. doi: 10.1093/nar/22.22.4673

Tillet, D., and Neilan, B. A. (2000). Xanthogenate nucleic acid isolation from cultured and environmental cyanobacteria. J. Phycol. 36, 251-258. doi: 10.1046/j.1529-8817.2000.99079.x

Valentine, D. L., Kessler, J. D., Redmond, M. C., Mendes, S. D., Heintz, M. B., Farwell, C., et al. (2010). Propane respiration jump-starts microbial response to a deep oil spill. Science 330, 208-211. doi: 10.1126/science.1196830

Vila, J., Nieto, J. M., Mertens, J., Springael, D., and Grifoll, M. (2010). Microbial community structure of a heavy fuel oil-degrading marine consortium: linking microbial dynamics with polycyclic aromatic hydrocarbon utilization. FEMS Microbiol. Ecol. 73, 349-362. doi: 10.1111/j.1574-6941.2010. 00902.x

Wilmotte, A., van der Auwera, G., and De Wachter, R. (1993). Structure of the 16S ribosomal RNA of the thermophilic cyanobacterium Chlorogloeopsis HTF (Mastigocladus laminosus HTF) strain PCC7518, and phylogenetic analysis. FEMS Microbiol. Lett. 317, 96-100. doi: 10.1016/0014-5793(93) 81499-P

Yakimov, M. M., Denaro, R., Genovese, M., Cappello, S., D’Auria, G., Chernikova, T. N., et al. (2005). Natural microbial diversity in superficial sediments of Milazzo Harbor (Sicily) and community successions during microcosm enrichment with various hydrocarbons. Environ. Microbiol. 7, 1426-1441. doi: 10.1111/j.1462-5822.2005.00829.x

Yakimov, M. M., Golyshin, P. N., Lang, S., Moore, E. R. B., Abraham, W.-R., Lönsdorf, H., et al. (1998). Alcanivorax borkumensis gen. nov., sp. nov., a new, hydrocarbon-degrading and surfactant-producing marine bacterium. Int. J. Syst. Bacteriol. 48, 339-348. doi: 10.1099/00207713-48-2-339

Yakimov, M. M., Timmis, K. N., and Golyshin, P. N. (2007). Obligate oil-degrading marine bacteria. Curr. Opin. Biotech. 18, 257-266. doi: 10.1016/j.copbio.2007.04.006

Yang, T., Nigro, L. M., Gutierrez, T., D’Ambrosio, L., Joye, S. B., Highsmith, R., et al. (2014). Pulsed blooms and persistent oil-degrading bacterial populations in the water column during and after the Deepwater Horizon blowout. Deep-Sea Res. II. doi: 10.1016/j.dsr2.2014.01.014. (in press).

Yu, Z., and Morrison, M. (2004). Comparisons of different hypervariable regions of rrs genes for use in fingerprinting of microbial communities by PCR-denaturing gradient gel electrophoresis. Appl. Environ. Microbiol. 70, 4800-4806. doi: 10.1128/AEM.70.8.4800-4806.2004

Conflict of Interest Statement: The authors declare that the research was conducted in the absence of any commercial or financial relationships that could be construed as a potential conflict of interest.

Received: 20 November 2013; paper pending published: 22 January 2014; accepted: 11 February 2014; published online: 27 February 2014.

Citation: Mishamandani S, Gutierrez T and Aitken MD (2014) DNA-based stable isotope probing coupled with cultivation methods implicates Methylophaga in hydrocarbon degradation. Front. Microbiol. 5:76. doi: 10.3389/fmicb.2014.00076

This article was submitted to Aquatic Microbiology, a section of the journal Frontiers in Microbiology.

Copyright (C) 2014 Mishamandani, Gutierrez and Aitken. This is an open-access article distributed under the terms of the Creative Commons Attribution License (CC BY). The use, distribution or reproduction in other forums is permitted, provided the original author(s) or licensor are credited and that the original publication in this journal is cited, in accordance with accepted academic practice. No use, distribution or reproduction is permitted which does not comply with these terms. 